\title{
Psychosurgery and the limbic system
}

\author{
DeSMOND Kelly \\ M.D., M.R.C.P., M.R.C.Psych. \\ St George's Hospital Medical School, Atkinson Morley's Hospital, \\ 31 Copse Hill, London, S.W.20
}

\begin{abstract}
Summary
The limbic system can be considered to consist of a central 'core' from which three well-defined circuits emerge. These are the Papez or medial circuit, the basolateral circuit and the defence reaction circuit. The functions of the primitive limbic brain are modulated by a higher order control-the frontal lobe. Emotional responses and physiological changes have been obtained by stimulation of fronto-limbic pathways and limbic circuits and these have been used for location of target sites in psychosurgery. For the relief of intractable depression and anxiety, lesions are generally made in the lower medial quadrant or posteroorbital part of the frontal lobe, where there is a concentration of fronto-limbic connections. In primary obsessional neurosis lesions at this site produce less satisfactory results, but these illnesses may be helped by lesions in the cingulate gyrus, which is part of the Papez circuit. Patients with epilepsy and aggressive outbursts may respond to temporal lobotomy, or to operations, such as amygdalotomy, where lesions are placed in the defence reaction circuit.
\end{abstract}

AFTER a long period of relative inactivity in the field of psychosurgery, there has recently been a resurgence of interest; this symposium is a reflection of that interest. It contains papers on the treatment of intractable depression, anxiety, obsessional neurosis and anorexia nervosa, and a paper on amygdalotomy for severely abnormal aggressive behaviour in eighteen patients, nearly all of whom had epilepsy.

The First International Congress on Psychosurgery was held in Lisbon in 1949 in honour of Professor Egas Moniz, and it was 21 years before the Second International Congress took place in Copenhagen in 1970. This had been stimulated by the introduction of new surgical techniques, and the Third World Congress took place only 2 years later, in Cambridge. Much of it was devoted to the relationship of the basic sciences with psychosurgery; and the two main reasons for the renewed interest have been the technological advances in the field of neurosurgery, and the greater understanding of physiological principles which underlie the various operations.
The limbic system

Most of the primitive cortex in mammals surrounds the brain stem and Broca called this large convolution 'the great limbic lobe'. The limbic system consists of the phylogenetically old rim of cortical tissue which encircles the inner part of the hemispheres of the brain. Limbic means 'forming a border around'; and this part of the cortex is involved in emotional behaviour.

In 1937 Papez wrote his classic paper entitled 'A Proposed Mechanism of Emotion.' He postulated that the anatomical basis of emotions was based in the hypothalamus, anterior thalamic nuclei, cingulate gyrus, hippocampus and their interconnections. He thought that this system constituted 'a harmonious mechanism which may elaborate the functions of central emotion, as well as participate in emotional expression'.

Yakovlev (1948) expanded the Papez concept to include the orbito-frontal cortex, insular and anterior temporal areas, together with their connections with the amygdala and the dorsomedial nucleus of the thalamus.

The limbic system may also affect pituitary secretion via hypothalamus and has been described as the 'visceral brain' by MacLean (1949). He suggested (MacLean, 1952) that the term 'limbic system' should include the limbic cortex* and subcortical cell stations with which it had primary connections. $\dagger$

\section{Limbic circuits}

(1) The Papez circuit (the medial limbic circuit of Livingston \& Escobar, 1973) passes from the septum via the cingulum bundle in the cingulate gyrus to hippocampus, and via fornix to mamillary body, via mamillo-thalamic tract to anterior thalamus, and finally via anterior thalamic radiation to cingulum bundle (Fig. 1).

* Limbic cortex. Cortex adjacent to the olfactory striae; pyriform area; hippocampal gyrus and hippocampus; paraspenial, cingulate and subcallosal gyri.

$\dagger$ Limbic subcortical cell stations. Amygdala, septal nuclei, hypothalamus, epithalamus, anterior thalamic nuclei, and parts of the basal ganglia. 


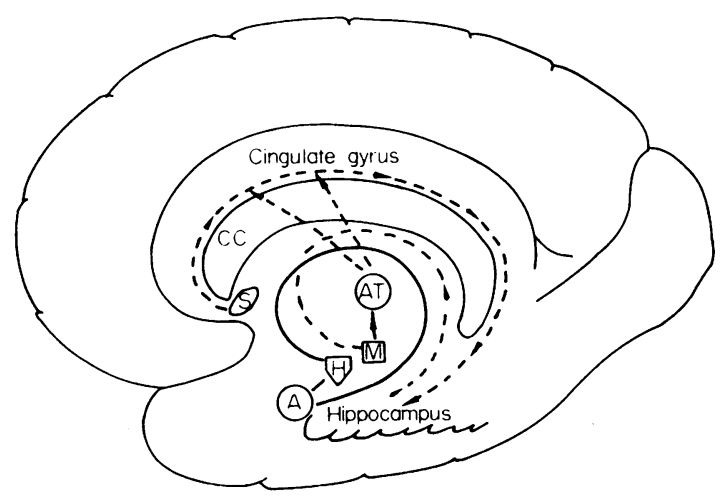

Fig. 1. The Papez circuit (broken line): passes via the cingulum bundle, in the cingulate gyrus, from septum (S) to hippocampus, and via fornix to mamillary body (M), via mamillothalamic tract to anterior thalamus (AT), via anterior thalamic radiations to cingulum bundle. The defence reaction circuit (solid line): passes from hypothalamus $(\mathrm{H})$ via stria terminalis to amygdala, and via amygdalo-fugal pathway to hypothalamus.

Stimulation of the Papez circuit in the anterior cingulate region of conscious patients has resulted in affective responses and physiological changes (Meyer et al., 1973). The latter have also been obtained in unconscious patients from stimulation of the cingulum bundle below and in front of the genu of the corpus callosum, and from the anterior cingulate region (Richardson, 1973; Kelly, 1972). Affective and autonomic responses have, therefore, been obtained from stimulation of this circuit (Fig. 2).

Yakovlev (1965) had noted that the cingulum is a bundle strategically situated to mediate activities of the limbic cortex to the entire forebrain.

The close association between emotional and autonomic pathways had already been emphasized by Livingston (1953), and autonomic responses are now being used to identify target sites in the cingulum bundle (Kelly, Richardson \& Mitchell-Heggs, 1973). This appears to be a fairly thin band of fibres in the vertical plane, as inserting the stimulating electrode a further $3 \mathrm{~mm}$ deep to the original site (Fig. 3) is sufficient to greatly diminish the physiological responses seen in Fig. 2.

Memory and the Papez circuit. Turner (1973) considers that the division of the limbic system into two parts, one for emotion and temperament and one for memory is justified, since the effects of ablation operations on the two parts are quite different. Anterior cingulate (Kelly \& MitchellHeggs, 1973), or posterior cingulate (Turner, 1973) lesions have no detectable effect on memory, whereas other structures in the Papez circuit, such as the hippocampus, fornix, mamillary bodies and mammillo-thalamic tract, are apparently concerned

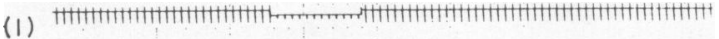

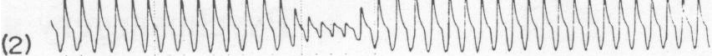

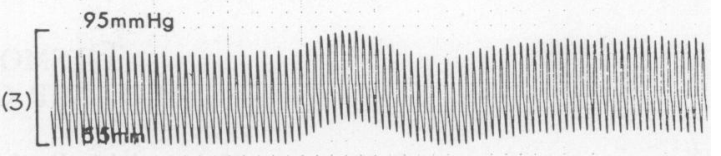

(4)

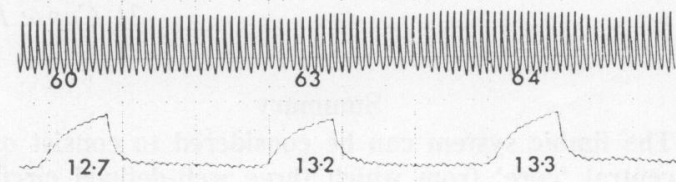

FIG. 2. Polygraph recording during stimulation of the anterior cingulate gyrus with $10 \mathrm{~V}$ under general anaesthesia showing the following autonomic changes:

Channel 1, time (sec), line depressed during stimulation.

Channel 2, respiration with decrease in amplitude.

Channel 3, blood pressure with increase in systolic and diastolic pressures.

Channel 4, heart rate increase with very slight increase in finger pulse amplitude after stimulation.

Channel 5, forearm blood flow $(\mathrm{ml} / 100 \mathrm{ml} / \mathrm{min})$ is increased. Finger pulse amplitude reflects changes in skin blood flow while forearm blood flow reflects predominantly changes in muscle.

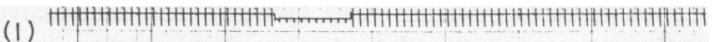

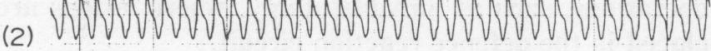

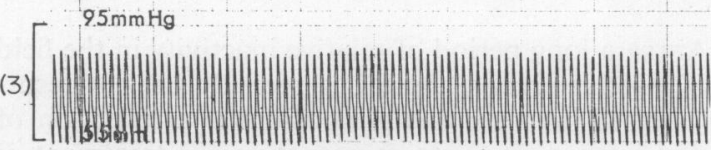

(4)

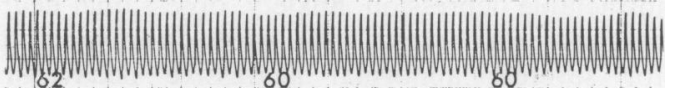

(5)

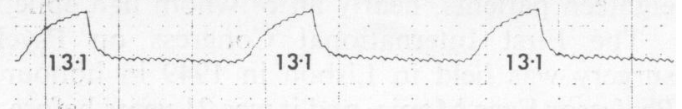

FIG. 3. Polygraph recording during stimulation of the anterior cingulate gyrus with $10 \mathrm{~V} 3 \mathrm{~mm}$ deep to site of stimulation in Fig. 2. By comparison the autonomic changes are considerably diminished. Restimulation of the original site restored autonomic changes in all four channels. Current spread from stimulation is thus fairly limited.

with recent and long-term memory (Turner, 1973).

(2) The basolateral circuit-orbital frontal-temporal-amygdalar-is closed via amygdalar-dorsomedial thalamic-frontal connections (bivingston, 
1969; Livingston \& Escobar, 1972). The anatomical relationship of this circuit to the brainstem, thalamus and neocortex, they consider to be distinct from the Papez circuit, and through its temporal connections it seems particularly concerned with the activities of the sensory-receptive and interpretive cortex (Livingston \& Escobar, 1972).

(3) The defence reaction circuit passes from hypothalamus via stria terminalis to amygdala, and via the amygdalofugal pathway back to hypothalamus (Fig. 1). With gradual increase in intensity of electrical stimulation of the hypothalamus, the conscious cat progresses from rest to looking alert, then becomes agitated and finally attacks in response to auditory and visual stimuli (Abrahams, Hilton \& Zbrozyna, 1960). This behavioural pattern is accompanied by changes in respiration, and an increase in heart rate and muscle blood flow, presumably to prepare the animal for 'fight' or 'flight'. Kaada (1972) has described amygaloid stimulation in animals leading to distinct patterns of 'flight', 'defence' and 'attack'.

Electrical stimulation of the rostral hypothalamus in conscious patients has caused marked discomfort with anxiety, tachycardia and peripheral autonomic effects (Heath \& Mickle, 1960).

Stimulation of the posteromedial hypothalamus in the waking state produced 'extreme horror' in conscious patients suffering from violent aggressive behaviour (Sano, Sekino \& Mayanagi, 1972). Hypothalamic stimulation was accompanied by tachycardia and an increase in blood pressure as well as an increase in plasma non-esterified fatty acids and growth hormone. Stereotactic lesions were subsequently made in an area from which the most marked signs of sympathetic discharge had been obtained, with a marked post-operative reduction in anxiety in 95\% of cases (Sano et al., 1972).

Stimulation of the amygdala has elicited a range of aggressive responses from ' $I$ feel I could get up and bite you', to uncontrolled swearing and physically destructive behaviour (Hitchcock \& Cairns, 1973). No pleasurable emotional feelings were identified by these workers in any of their patients during stimulation or coagulation of the amygdala. Three of Hitchcock's patients expressed a desire to get away: 'The significance of this, which might be termed "flight" behaviour, in relation to known patterns following amygdaloid stimulation in animals in whom distinct patterns of flight, defence and attack responses have been observed (Kaada, 1972), is of some interest.' Hitchcock (1973) uses emotional responses for target location in conscious patients, and has also noted hormonal changes following amygdalotomy, while autonomic changes have been observed following amygdaloid stimulation in unconscious patients (Kelly, 1972).

\section{Limbic core and connections}

(A) The medial forebrain bundle. 'The medial forebrain bundle (MFB) and its continuation as the cingulum (Fig. 4) may be considered to be to the limbic system what the internal capsule is to the outer convexity of the brain' (MacLean, 1958).

The medial forebrain bundle runs forward from the 'limbic midbrain area' (vide infra) as an ascending pathway to various parts of the hypothalamus and then divides into two large streams. One branch turns medially to the septum to converge with fibres descending from the medial olfactory tract, and feeds into the cingulum and Papez circuit (MacLean, 1958).

The other branch turns laterally into the region of the amygdala where it converges with descending fibres from the lateral olfactory tract. From amygdala fibres run into the limbic cortex of the fronto-temporal region.

The medial forebrain bundle also contains a descending system of fibres.

(B) Septo-hypothalamo-mesencephalic continuum. Nauta (1958) emphasized the extension of the limbic system into a paramedian zone of the midbrain which he referred to as the 'limbic midbrain area'

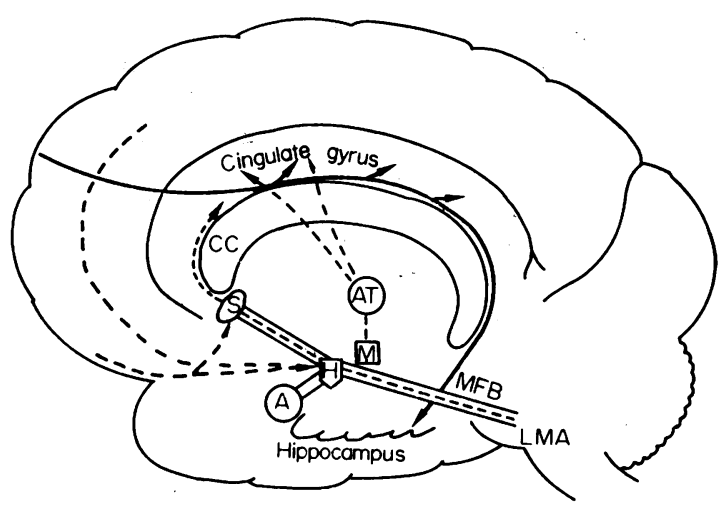

FIG. 4. 'Limbic core' and connections. The medial forebrain bundle (MFB) runs from the limbic midbrain area (LMA) to mamillary body (M) and hypothalamus (H), where one branch turns laterally to amygdala (A) and the other medially to septum (S). This system constitutes the septo-hypothalamo-mesencephalic continuum (SHMC) of Nauta and may be considered to be the central 'limbic core' of the limbic system.

The SHMC or 'limbic core' projects: (a) via mamillothalamic tract to anterior thalamic nucleus (AT) and cingulate gyrus; (b) via medial forebrain bundle which contains serotonin fibres ascending directly to cingulate gyrus (dashed line) round the corpus callosum (CC).

The frontal lobe projects to SHMC: (a) from orbito-frontal cortex to (i) septum, (ii) lateral hypothalamus; (b) from dorsal frontal convexity to lateral and dorsal hypothalamus; (c) from dorsal frontal convexity via cingulate gyrus to hippocampus. 
(Fig. 4). He described a fairly well-defined subcortical continuum that extends from the septal region and hypothalamus into the limbic midbrain area, the 'septo-hypothalamo-mesencephalic continuum'. This highly differentiated subcortical apparatus is known to be centrally involved in the regulation of endocrine and visceral-effector mechanisms and Nauta (1973) speculated that its functional patterns express themselves in moods and behavioural motivations.

An interpretation of Nauta's work is that the septo-hypothalamo-mesencephalic continuum may be thought of as the central 'limbic core' with circuits extending from it. The septo-hypothalamomescencephalic continuum receives downward discharge from limbic structures, especially hippocampus and amygdala via fornix, medial forebrain bundle and stria terminalis, and projects to these structures by two main pathways (Fig. 4); (a) via mamillo-thalamic tract and anterior thalamic nucleus and cingulate cortex for the dorsal convexity field, and via dorso-medial nucleus for orbitofrontal cortex (not shown in Fig. 4); (b) via medial forebrain bundle, which contains among other components, serotonin fibres originating in midbrain tegmentum and apparently ascending directly to the cingulate gyrus.

The medial forebrain bundle also contains noradrenaline-carrying fibres, presumably arising from neurones lying outside that area in more lateral regions of the brainstem tegmentum (Nauta, 1973). Lewis \& Shute (1967) showed that adrenergic and cholinergic fibre systems supply the limbic cortex and Paasonen, MacLean \& Gairman (1957) demonstrated high concentrations of serotonin in the limbic system. For a review of catecholamine containing pathways see Crow (1973a, b). Nauta (1973) considers that it is only natural to assume that any central nervous mechanism that has efferent connections with either the limbic system or the septohypothalamo-mesencephalic continuum, or both, is potentially a modulator of viscero-endocrine function and affect.

The septo-hypothalamo-mesencephalic continuum is interconnected by distinct intrinsic circuitry (Nauta, 1958) into which lead afferent systems from the amygdala and hippocampus as well as from spinal cord and brainstem, and from which emerge channels to the hypophysial complex and visceral motor system.

\section{Fronto-limbic connections}

Adey \& Meyer (1952) demonstrated that the frontal cortex projects to the 'limbic' cortex (gyrus cinguli, retrosphlenial cortex and parahippocampal gyrus). Lesions involving the medial surface of the frontal lobe produced fibre degeneration caudal- wards in the cingulum bundle as far as the presubiculum and entorhinal area of the parahippocampal gyrus. This fronto-limbic pathway (Fig. 4, pathway c) was confirmed by later studies (Nauta, 1964; Pandya \& Kuypers, 1969; Jones \& Powell, 1970) although none could confirm a connection with the entorhinal area. Nauta (1964) suggested that this association system in the monkey originates mainly in the dorsal part of frontal convexity, perhaps also from the medial frontal cortex and is distributed to the cortex of the cingulate gyrus and presubiculum and via the latter almost certainly with the circuitry of the hippocampus.

A second fronto-limbic pathway has been demonstrated more recently by Van Hoesen, Pandya \& Butters (1971). It originates in the caudal part of the cortex covering the orbital surface of the frontal lobe and extends caudalwards over a ventral route to the entorhinal area immediately behind the olfactory cortex of the uncus. The entorhinal area has massive projections to the hippocampus, hence the orbitofrontal cortex can affect the neural mechanisms of the hippocampus.

Frontal lobe projections to the septo-hypothalamomesencephalic continuum: Only the frontal lobe is known to project directly to the septohypothalamo-mesencephalic continuum, from two widely separate areas (Nauta, 1973): (1) from orbito-frontal cortex through substriatal grey matter (substantia innominata) to, (i) septum, (ii) lateral hypothalamus (Fig. 4); (2) from dorsal region of frontal convexity to lateral and dorsal hypothalamus (Fig. 4), and nucleus centralis tegmenti superior.

The frontal cortex, therefore, both monitors and modulates limbic mechanisms (Nauta, 1971).

\section{Eating and the limbic system}

It is known from animal experiments that damage to the ventromedial hypothalamus can result in overeating, whereas lateral hypothalamic lesions produce aphagia. Electrical stimulation of the lateral hypothalamus can elicit eating or drinking. The hypothalamic stimulation which elicits appetitive motivation is also 'rewarding' (Rolls, 1972). All sites from which eating, drinking, or sexual behaviour can be elicited are 'self-stimulation' sites. Self-stimulation near the medial forebrain bundle between the preoptic area and the mid-brain, including hypothalamic sites, is associated with hyperactivity. Stimulation of the amygdala in rats can be either 'rewarding' or 'aversive', depending on which part is stimulated. It has been shown recently that electrical stimulation of the lateral hypothalamus of the rat which produces eating, drinking or 'reward' produces firing of neurones of the amygdala and pyriform cortex, which are involved in these functions (Rolls, 1972). It is known that electrical stimulation of the amyg- 
dala in the cat can activate hypothalamic neurones through either the stria terminalis or ventral pathway (Fig. 1). It is not known which of these pathways is involved when lateral hypothalamic stimulation results in firing of neurones in the amygdala.

Lateral hypothalamic stimulation also activates brain stem neurones (Rolls, 1971), and stimulation of 'rewarding' sites in the tegmental region of the pons and lateral hypothalamus produces firing of neurones in the lateral and medial pre-frontal cortex. In the monkey, orbito-frontal cortex unit firing has been observed following stimulation of the amygdala and lateral hypothalamus, among other sites which produce 'reward'. It is concluded that amygdala and pre-frontal cortex are higher order centres concerned with eating and 'reward' (Rolls, 1972; Rolls \& Cooper, 1973).

This work on animals may give some neurophysiological insight into the improvement in anorexia nervosa following lower medial quadrant lesions of the frontal lobe in man (Crisp \& Kalucy, 1973).

\section{Summary of anatomical considerations}

Evidence has been presented which suggests that the limbic system has a central 'core' from which three well-defined circuits emerge. These are, (i) the Papez or medial circuit, (ii) the basolateral circuit and (iii) the defence reaction circuit (Fig. 1). Emotional responses and physiological changes have been obtained from stimulation of these circuits in conscious patients, the latter persisting under general anaesthesia. Both emotional responses and physiological changes have been used as an aid to target location during operations designed to interrupt some of these circuits.

The phylogenetically primitive limbic brain is monitored and modulated by a higher order control - the frontal lobe. There are two main projections from the frontal lobe to the 'limbic core', (i) from the dorsal convexity and (ii) from the orbito-frontal cortex (Fig. 4). There is a third main fronto-limbic connection from the dorsal convexity to the cingulate gyrus in the Papez circuit. From this it can be seen that there is a concentration of fronto-limbic connections in three main areas; (a) the lower medial quadrant of the frontal lobe, (b) the fibre tracts overlying the posterior orbital cortex and (c) the cingulate gyrus which contains the cingulum bundle. The first two target zones converge at their posterior end and overlap one another.

For the relief of intractable depression, anxiety and obsessional neurosis the main areas operated on are, (a) the lower medial quadrant, (b) frontoorbital and (c) cingulate regions. For severely abnormal aggressive behaviour operations have been carried out on various parts of the defence reaction circuit.
The limbic system is extremely complicated and only well defined circuits and connections have been considered.

Psychiatric syndromes and target sites for operations

Frontal lobe: fronto-limbic connections

The orginal frontal lobotomy of Freeman \& Watts (1942) massively divided fronto-limbic connections in a plane anterior to the tip of the lateral ventricle and involved all four quadrants of the frontal lobe. It fell into disrepute because this extensive lesion was often associated with impairment of frontal lobe function, leading to apathy with flatness of affect, or euphoria with disinhibition, or impaired social awareness and a relative disregard for the feelings of others, indicative of a major personality change. When the operation was first introduced it was performed mainly on patients with chronic schizophrenia, for which treatment was very limited at that time. With the introduction of insulin coma, ECT, and later the phenothiazines, the frequency of standard lobotomy for schizophrenia diminished.

Modifications of the original Freeman \& Watts operation concentrated on the medial part of the frontal lobe, and include bimedial, rostral and the modified prefrontal leucotomy of Harvey Jackson. The operations all concentrate on the lower medial quadrant, and divide both of the frontal lobe projections to the septo-hypothalamo-mesencephalic continuum (central 'limbic core'). The historical development of these modifications and the results of this type of operation and its different effects are considered by Turner (1973). Lower quadrant frontal lobotomy with the plane of section in front of the anterior horn of the lateral ventricle via a lateral approach gives good results in depression (Turner, 1973). This approach is similar to that used by Harvey Jackson (1954) who also obtained good results in depression and anxiety states (Kelly et al., 1972). Rostral leucotomy employs a superior approach (McKissock, 1959) and gives similar results in anxiety and depression, but in all three operations the results in obsessional neurosis are not as satisfactory. Good results have been obtained, even in severe obsessional neurosis with an early age of onset, with limbic leucotomy (Kelly \& MitchellHeggs, 1973), in which a combination of small lower medial quadrant and anterior cingulate lesions are placed by a stereotactic technique (Richardson, 1973). With this operation the results in anxiety and depression are comparable to more extensive freehand operations of the rostral and Harvey Jackson type (Kelly et al., 1973).

Para-median lobotomy is considered by Turner (1973) to benefit patients with anxiety and impulsive behaviour, usually of an aggressive nature. Young 
patients with obsessional neurosis do not usually respond to this operation, however.

The other main target site in the frontal lobe is the posterior orbito-frontal region. Le Gros Clark \& Meyer (1950) demonstrated fibres which pass from Area 13 in the orbital cortex to the hypothalamus. The orbital undercut of Scoville was modified by Knight (1964), who subsequently developed a stereotactic operation restricted to the last $2 \mathrm{~cm}$ of a $6 \mathrm{~cm}$ restricted orbital undercutting incision. Autonomic responses can be obtained from Area 13, and Smith (1945) and Livingston et al. (1948a) obtained respiratory and vascular responses from a continuous zone of cortex extending from the anterior cingulate gyrus to the posterior orbital surface in the monkey. Lesions in these areas produced loss of fear, diminished aggression and increased tameness (Ward, 1948).

In patients, pre-leucotomy stimulation of the orbital part of the frontal lobe also resulted in changes in respiration and blood pressure and was sometimes accompanied by intense emotion (Livingston et al., 1948b). Knight (1973) describes his extensive experience with stereotactic tractotomy in this symposium, with very good results in depression and anxiety but less satisfactory results in obsessional neurosis. 'There does seem, however, to be a relatively small group of cases characterized by pure obsessional symptoms, without depression, of early age of onset, which has a bad prognosis.' The target site of stereotactic tractotomy overlaps the lower medial quadrant target site of limbic leucotomyalthough the approaches are from different anglesthe plane of the lesion is almost horizontal in the former (Knight, 1973; Fig. 3), and vertical in the latter (Richardson, 1973; Fig. 2).

In the lower medial quadrant, and the posteriororbital part, of the frontal lobe there is a concentration of fronto-limbic connections (Fig. 4) and it is tempting to speculate that it is the division of these that causes the beneficial effects of stereotactic tractotomy and lower medial quadrant lesions.

It can be seen from Figs. 1 and 4 that limbic connections are concentrated in two main areas: in the lower medial quadrant and posterior-orbito-frontal lobe and in the anterior cingulate gyrus. It is remarkable how these two widely separated target zones yield very similar autonomic responses, and this suggests that fibres may be stimulated which mediate similar functional pathways (Figs. 2 and 5).

It is noteworthy that moving from these target sites by as little as 3-4 mm may be sufficient to almost completely abolish the responses. The symmetry of autonomic response pattern from either side of the brain in the lower medial quadrant of the frontal lobe and anterior cingulate gyrus is also impressive. Recently, increase in cortisol secretion has been

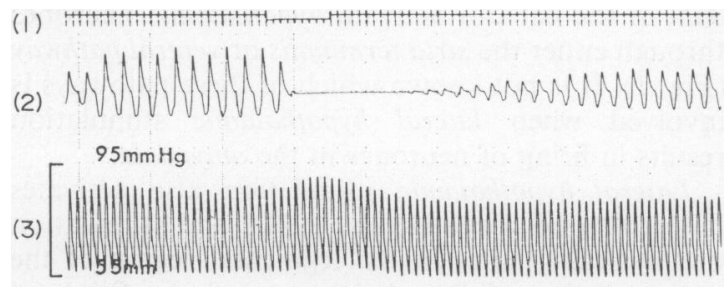

(4)

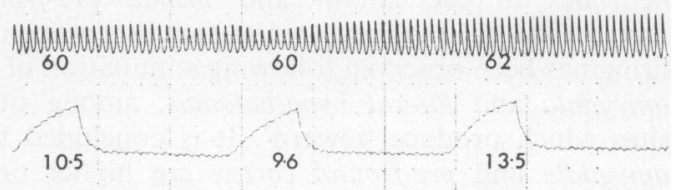

FIG. 5. Polygraph recording during stimulation of the lower medial quadrant of the frontal lobe with $10 \mathrm{~V}$ under general anaesthesia showing autonomic changes which in some cases are similar to those shown in Fig. 2. Channel 1, time (sec), line depressed during stimulation.

Channel 2, respiration with apnoea continuing after stimulation.

Channel 3, blood pressure with increase in systolic and diastolic pressures.

Channel 4, heart rate increase after stimulation with cutaneous vaso-constriction during stimulation with subsequent cutaneous vasodilatation.

Channel 5, forearm blood flow $(\mathrm{ml} / 100 \mathrm{ml} / \mathrm{min})$ with vaso-constriction during stimulation with subsequent vasodilatation (muscle).

found to result from electrical stimulation of both these sites, particularly the former, during limbic leucotomy (Jenkins \& Frankel, 1973). The maximum increase in cortisol was $375 \%$.

\section{Papez circuit: cingulate gyrus}

Anterior cingulate lesions interrupt the other main fronto-limbic pathway (Fig. 4) as well as interrupting one of the main limbic circuits. This can reduce pathological psychiatric symptoms, without effecting normal affective responses (Case 3; Kelly \& MitchellHeggs, 1973). Operations on the Papez circuit have concentrated on the anterior cingulate gyrus. Fulton suggested this site at a meeting of the Society of British Neurological Surgeons in 1947 and later Sir Hugh Cairns performed the first open cingulectomy. Lewin (1961) reported the findings on fifty-two patients who had undergone this operation; and found it to be particularly beneficial in patients suffering from obsessional disorders. He considered that the operation may interrupt a 'reverberating circuit', formed by hippocampus, fornix, mammillary bodies, anterior nucleus of thalamus, and cingulate gyrus, i.e. the Papez circuit.

Meyer et al. (1973) stimulated the anterior cingulate region and obtained affective responses and physiological changes in psychiatric patients. Sub- 
sequently, anterior cingulotomy resulted in significant overall improvement in a variety of psychiatric disorders. Ballantine et al. (1967) and Brown \& Lighthill (1968) also obtained good results with lesions placed by stereotaxis in the anterior cingulate gyrus.

\section{The baso-lateral and defence reaction circuits}

Personality disorders associated with epilepsy, and aggressive outbursts or 'uncontrollable rage, usually of a particular kind ushered in by experiences of sudden fear, panic or stark terror' respond to temporal lobotomy (Turner, 1973). The site of operation is at, or just behind the uncus of the hippocampus and in, or just behind the amygdaloid nucleus. Fear is abolished if ictal, but chronic anxiety is generally not helped. Hitchcock \& Cairns (1973) and Balasumramaniam \& Ramamurthi (1968) have also found stereotactic amygdalotomy beneficial in modifying aggressive behaviour associated with epilepsy. In both these operations lesions are being placed in the defence reaction circuit. In addition to operations on the amygdala, other workers have obtained clinical improvement from other sites in the defence reaction circuit. In patients suffering from severely disturbed destructive behaviour, Sano et al. (1972) operated on the postero-medial hypothalamus, and Burzaco (1973) on the stria terminalis.

\section{Psychosurgery for anorexia nervosa}

Crisp \& Kalucy (1973) describe in this symposium the effects of leucotomy on four patients with primary anorexia nervosa, and conclude that in this syndrome leucotomy has and will continue to have a limited but very definite place in treatment. The detailed reports illustrating the relationship between weight gain and psychological change are of considerable interest, and the application of the repertory grid technique to the evaluation of changes in attitude following leucotomy adds another dimension to the methods now available for postoperative assessment. The inability of one of their patients to tolerate the psychological consequences of leucotomy has important implications, and underlines the difficulty in predicting the effect of the operation in very neurotic vulnerable personalities. The complex relationship between eating and 'reward'and the pathways involved-are only now beginning to be explored in depth.

\section{Conclusion}

There still appears to be a place for psychosurgery, 'the challenge being to precisely locate the indications within the total field of psychiatry' (Bridges \& Bartlett, 1973). It must be remembered that affective disorders carry a $15 \%$ risk of death from suicide, and psychosurgery can relieve intolerable suffering and improve the quality of life when all other treatments have failed. The main indications are intractable depression, anxiety and obsessional neurosis. Some patients with schizophrenia, and very rarely patients with anorexia nervosa, can also be helped. Operations for seriously aggressive behaviour, such as posterior cingulectomy and paramedian frontal lobotomy (Turner, 1973), amygdalotomy and postero-medial hypothalamotomy are more controversial, and experience in this country is, so far, limited.

The various target sites chosen by different neurosurgeons can be understood in terms of lesions placed in different parts of well defined fronto-limbic connections and limbic circuits.

Technical advances in neurosurgery are likely to lead to a decrease in the number of 'free hand' operations and an increase in the use of stereotactic techniques. Far more sophisticated methods of psychiatric, psychological, biochemical and psychophysiological assessment are now available, and will be increasingly used.

Progress is likely to come from close multidisciplinary co-operation, as indicated in this symposium, and with increasing sophistication in this field, it would probably be wise for this highly specialized type of treatment to be concentrated in a relatively few centres as suggested by Bridges \& Bartlett (1973). It is hoped that there will come a time when psychosurgery is no longer required for the tiny minority of psychiatric patients for whom, at present, no other type of treatment is effective. Meanwhile, the major question is, if all else fails, which patients are suitable for psycho-surgery, and which operation should they have?

\section{References}

Abrahams, V.C., Hilton, S.M. \& Zbrozyna, A. (1960) Active muscle vasodilatation produced by stimulation of the brain stem: its significance in the defence reaction. Journal of Physiology, 154, 491.

AdEY, W.R. \& MeYer, M. (1952) Hippocampal and hypothalamic connections of the temporal lobe in the monkey. Brain, 75, 358.

Balasumramaniam, V. \& Ramamurthi, B. (1968) Stereotaxic amygdalotomy. Proceedings of the Australian Association of Neurologists, 5, 277.

Ballantine, H.T., Cassidy, W.L., Flanagan, N.B. \& MARINO, R. (1967) Stereotactic anterior cingulotomy for neuropsychiatric illness and intractable pain. Journal of Neurosurgery, 26, 488.

Bridges, P.K. \& Bartlett, J.R. (1973) The work of a psychosurgical unit. Postgraduate Medical Journal, 49, 855.

Brown, H.M. \& Lighthill, J.A. (1968) Selective anterior cingulotomy: a psychosurgical evaluation. Journal of Neurosurgery, 29, 513.

Burzaco, J.A. (1973) Fundus striae terminalis, an optimal target in sedative stereotactic surgery. In: Surgical Approaches in Psychiatry (Ed by Laitinen and Livingston), p. 135. Medical and Technical Publishing Co. Ltd. Lancaster. 
Clark, W.E. Le Gros \& Meyer, M. (1950) Anatomical relationships between the cerebral cortex and hypothalamus. British Medical Bulletin, 6, 341.

CRISP, A.H. \& KALUCY, R.L. (1973) The effect of leucotomy in intractable adolescent weight phobia (primary anorexia nervosa). Postgraduate Medical Journal, 49, 883.

CROW, T.J. (1973a) Catecholamine-containing neurones and electrical self-stimulation: a review of some data. Psychological Medicine, 2, 414.

Crow, T.J. (1973b) Catecholamine-containing neurones and electrical self-stimulation: a theoretical interpretation and some psychiatric implications. Psychological Medicine, 3, 66.

Freeman, W. \& Watts, J. (1942) Psychosurgery. Charles C. Thomas, Springfield. Illinois.

Heath, R.G. \& Mickle, W.A. (1960) Evaluation of seven years experience with depth electrode studies in human patients. In: Electrical Studies on the Unanesthetized Brain (Ed. by Ramey and O'Doherty). New York.

Hitchcock, E. \& Cairns, V. (1973) Amygdalotomy. Postgraduate Medical Journal, 49, 894.

JACKSON, H. (1954) Leucotomy-a recent development. Journal of Mental Science, 100, 62.

Jenkins, J. \& Frankel, R. (1973) Personal communication.

JoNES, E.G. \& Powell, T.P.S. (1970) An anatomical study of converging sensory pathways within the cerebral cortex of the monkey. Brain, 93, 793.

KAADA, B. (1972) Stimulation and regional ablation of the amygdaloid complex with reference to functional representations. In: The Neurobiology of the Amygdala (Ed. by B. E. Eleftheriou), p. 205. Plenum Press, New York.

Kelly, D. (1972) Physiological changes during operations on the limbic system in man. Conditional Reflex, 7, 127.

Kelly, D. \& Mitchell-Heggs, N. (1973) Stereotactic limbic leucotomy: a follow-up study of thirty patients. Postgraduate Medical Journal, 49, 865.

Kelly, D., Richardson, A. \& Mitchell-Heggs, N. (1973) Stereotactic limbic leucotomy: neurophysiological aspects and operative technique. British Journal of Psychiatry, 123, 133.

Kelly, D., Richardson, A., Mitchell-Heggs, N., GreenUP, J., Chen, C. \& Hafner, R.J. (1973) Stereotactic limbic leucotomy: a preliminary report on forty patients. British Journal of Psychiatry, 123, 141.

Kelly, D., Walter, C.J.S., Mitchell-Heggs, N. \& SARGANT, W. (1972) Modified leucotomy assessed clinically, physiologically and psychologically at six weeks and eighteen months. British Journal of Psychiatry, 120, 19.

KNIGHT, G. (1964) The orbital cortex as an objective in the surgical treatment of mental illness. British Journal of Surgery, 51, 114.

KNIGHT, G. (1973) Further observations from an experience of 660 cases of stereotactic tractotomy. Postgraduate Medical Journal, 49, 845.

LEWIN, W. (1961) Observations on selective leucotomy. Journal of Neurology, Neurosurgery and Psychiatry, 24, 37.

LEWIS, P.R. \& SHUTE, C.C.D. (1967) The cholinergic limbic system: projections to hippocampal formation, medial cortex, nuclei of the ascending cholinergic reticular system, and the subfornical organ and supra-optic crest. Brain, $90,521$.

Livingston, K.E. (1953) Cingulate cortex isolation for the treatment of psychoses and psychoneuroses. Research Publications of the Association for Research in Nervous and Mental Disease, 31, 374.

Livingston, K.E. (1969) The frontal lobes revisited: the case for a second look. Archives of Neurology, 20, 90.

Livingston, K.E. \& Escobar, A. (1972) The continuing evolution of the limbic system concept. In: Psychosurgery (Ed. by Hitchcock, Laitinen and Vaernet), p. 25. Charles C Thomas, Springfield, Illinois.
Livingston, R.B., Chapman, W.P., Livingston, K.E. \& KRAINTZ, L. (1948a) Stimulation of the orbital surface of man prior to frontal lobotomy. Research Publications of the Association for Research in Nervous and Mental Disease, 27, 421.

Livingston, R.B., Fulton, J.F., Delgado, J.M.R., SaChs, F. JR, BRendler, S.J. \& DAvis, G.D. (1948b) Stimulation and regional ablation of orbital surface of frontal lobe. Research Publications of the Association for Research in Nervous and Mental Disease, 27, 405.

MacLean, P.D. (1949) Psychosomatic disease and the 'visceral brain'. Recent developments bearing on the Papez theory of emotion. Psychosomatic Medicine, 11, 338.

MacLean, P.D. (1952) Some psychiatric implications of physiological studies on frontotemporal portion of the limbic system (visceral brain). Electroencephalography and Clinical Neurophysiology, 4, 407.

MacLean, P.D. (1958) Contrasting functions of limbic and neocortical systems of the brain and their relevance to psychophysiological aspects of medicine. American Journal of Medicine, 25, 611 .

Meyer, G., McElhany, M., Martin, W. \& McGraw, C.P. (1973) Stereotactic cingulotomy. In: Surgical Approaches in Psychiatry (Ed. by Laitinen and Livingston), p. 39. Medical and Technical Publishing Co. Ltd, Lancaster.

McKissock, W. (1959) Discussion on psychosurgery. Proceedings of the Royal Society of Medicine, 52, 206.

NaUTA, W.J.H. (1958) Hippocampal projections and related neural pathways to the midbrain in the cat. Brain, 81, 319.

NauTA, W.J.H. (1964) Some efferent connections of the prefrontal cortex in the monkey. In: The Frontal Granular Cortex and Behaviour (Ed. by J. M. Warren and K. Akert), p. 397. McGraw-Hill, New York.

NAUTA, W.J.H. (1971) The problem of the frontal lobe: a reinterpretation. Journal of Psychiatric Research. 8, 167.

NaUta, W.J.H. (1973) Connections of the frontal lobe with the limbic system. In: Surgical Approaches in Psychiatry (Ed. by Laitinen and Livingston), p. 303. Medical and Technical Publishing Co. Ltd, Lancaster.

Paasonen, M.K., Maclean, P.D., \& Gairman, N.J. (1957) 5-Hydroxytryptamine (serotonin, enteramine) content of structures of the limbic system. Journal of Neurochemistry, 1, 326.

PANDYA, D.N. \& KuYPers, H.G.J.M. (1969) Cortico-cortical connections in the rhesus monkey. Brain Research, 13, 13.

PAPEZ, J.W. (1937) A proposed mechanism of emotion. Archives of Neurology and Psychiatry, 38, 725.

RichaRdSON, A. (1973) Stereotactic limbic leucotomy: surgical technique. Postgraduate Medical Journal, 49, 860.

RoLLS, E.T. (1971) Involvement of brainstem units in medial forebrain bundle self-stimulation. Physiology and $\mathrm{Be}$ havior, 7, 297.

Rolls, E.T. (1972) Activation of amygdaloid neurones in reward, eating and drinking elicited by electrical stimulation of the brain. Brain Research, 45, 365.

Rolls, E.T. \& CoOPER, S.J. (1973) Activation of neurones in the prefrontal cortex by brain-stimulation reward in the rat. Brain Research (in press).

Sano, K., Sekino, H. \& Mayanagi, Y. (1972) Results of stimulation and destruction of the posterior hypothalamus in cases with violent, aggressive or restless behaviors. In: Psychosurgery (Ed. by Hitchcock Laitinen and Vaernet), p. 57. Charles C. Thomas, Springfield, Illinois.

Smith, W.K. (1945) The functional significance of the rostral cingular cortex as revealed by its responses to electrical excitation. Journal of Neurophysiology, 8, 241.

Turner, E. (1973) Custom psychosurgery. Postgraduate Medical Journal, 49, 834. 
Van Hoesen, G.W., Pandya, D.N. \& Butters, N. (1971) Cortical efferents to the entorhinal cortex of the rhesus monkey. Science, 175, 1471.

WARD, A.A. JR (1948) The anterior cingular gyrus and personality. Research Publications of the Association of Nervous and Mental Disease, 27, 438.
Yakovlev, P.I. (1948) Motility, behavior and the brain: stereodynamic organization and neural co-ordinates of behavior. Journal of Nervous and Mental Diseases, 107, 313-

Yakovlev, P.I. (1965) Personal communication to Ballan. tine et al., 1967.

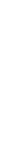

